MIDPI

MOL2NET, International Conference Series on Multidisciplinary Sciences

\title{
sciforum
}

\section{Structural characterization of the FcDFR1-Dihydroflavonols interactions using Molecular Dynamic Symulations}

\author{
Parra-Palma, C. ${ }^{a, b}$, Ramos, $P^{a}$., Moya-León, M.A. ${ }^{a}$
}

a Laboratorio de Fisiología Vegetal y Genética Molecular, Instituto de Ciencias Biológicas, Universidad de Talca

b (cparra@utalca.cl) Programa de Doctorado en Ciencias Mención Ingeniería Genética Vegetal, Universidad de Talca

Graphical Abstract

Abstract.

Dihydroflavonol 4-reductase (DFR) is a pivotal enzyme in the flavonoid biosynthesis pathway catalyzing the last common step that leads to anthocyanins and proanthocyanidins. DFR promotes the reduction of three

Insert grafical abstract dihydroflavonols: dihydrokaempferol (DHK), dihydroquercetin (DHQ) and figure here dihydromyricetin (DHM) to leucoanthocyanidins. These substrates differ only in the number of hydroxyl groups on the $\mathrm{B}$ phenyl ring: only one, two or three to DHK, DHQ and DHM respectively. Recently, a new variant of DFR (DFR1), which showed an unusual preference for only DHK was identified in strawberry, meanwhile DFR2 can convert any of the three dihydroflavonols. A region of 26 amino acid residues could be relevant to identify the substrates, proposed as the binding pocket of B phenyl ring of dihydroflavonols, where an asparagine residue could be critical. To determine the importance of these differences in both proteins, a characterization at structural level by homology model methodology was carried out to FcDFR1 and FcDFR2 from the Chilean strawberry (Fragaria chiloensis). Additionally, by molecular dynamics simulation we identify differences in substrate binding mode of the proteins with DHK and DHQ. Phylogenetic analyses grouped FcDFR1 and FcDFR2 into separate clades. FcDFR1 and FcDFR2 sequences consist of 341 and 350 amino acid residues respectively, and share $78.6 \%$ sequence identity. The most important differences were found in the region that is important for substrate identification. FcDFR1 and FcDFR2 structures were obtained through comparative modeling, showing a RMSD of $2.39 \AA$. Regarding protein-ligand interactions, in FcDFR2 a strong and stable interaction between Asn133 and the 3 '-OH group on ring B of DHQ was determined by molecular dynamics simulations, but not in FCDFR1, where the equivalent residue is Ala135. In contrast, DHK without 3'-OH group could be transformed by both enzymes as stable interactions were determined. The data provides an explanation of why DFR1 could interact with DHK and not with $\mathrm{DHQ}$.

\section{Introduction (optional)}

Diversity of colors in flowers and fruits is largely due to anthocyanin pigments. In the receptacle of white chilean strawberry fruit the main anthocyanin pigment acumulated is cyanidin-3-glucoside. On 
the other hand, in the red fruit of $F$. $x$ ananassa there is a higher acumulation of pelargonidin-3glucoside (Simirgiotis et al., 2009). The accumulation of different types of anthocyanins can be due to: a) the negative regulation of the expression of $\mathrm{F}^{\prime} \mathrm{H}$ in late stages of the fruit; or b) to the presence of a set of DFRs having different substrate specificity.

Dihydroflavonol 4-reductase (DFR) is a pivotal enzyme in the flavonoid biosynthesis pathway catalyzing the last common step that leads to anthocyanins and proanthocyanidins (Beld et al., 1989).

DFR promotes the reduction of three dihydroflavonols: dihydrokaempferol (DHK), dihydroquercetin (DHQ) and dihydromyricetin (DHM) to leucoanthocyanidins (Johnson et al., 1999; Schwinn et al., 2014). These substrates differ only in the number of hydroxyl groups on the B phenyl ring: DHK only one, DHQ two, DHM three. The substrate specificity of the DFRs was found to play a crucial role in determination of type of anthocyanins, thereby determining the color of the pigment.

Recently, a new variant of DFR (DFR1), which showed an unusual preference for only DHK was identified in strawberry, meanwhile DFR2 can convert any of the three dihydroflavonols (Miosic et al., 2014). A region of 26 amino acid residues could be relevant to identify the substrates, proposed as the binding pocket of $\mathrm{B}$ phenyl ring of dihydroflavonols, where an asparagine residue could be critical (Johnson et al., 2001; Petit et al., 2007).

We analyzed FcDFR1 and FcDFR2 isolated from the Chilean white strawberry fruit (F. chiloensis spp. chiloensis) at sequence and structural level to identify differences in substrate binding (DHK and DHQ). These differences could explain the preference for a particular substrate, leading to $F$. chiloensis fruit accumulating a particular type of anthocyanins.

\section{Conclusions (optional)}

The deduced amino acid sequences of FcDFR1 and FcDFR2 have 78\% similarity. Both proteins displayed similar 3D structures, and share the characteristic motifs of this type of enzymes: the cofactor-binding and the substrate-binding sites.

Minor differences in FcDFR1 and FcDFR2 in the region composed of 26 residues seems to be crucial for substrate selectivity: Asn133 in FcDFR2 interacts with the 3'OH of DHQ.

Molecular Dynamic simulations point out the residues involved in H-bonds formation with the $\mathrm{OH}$ groups of dihydroflavonols which are relevant for substrate binding, but does not necessarily determine substrate specificity.

The residues present in the region described for substrate selectivity in both proteins display differences in their physicochemical properties that could explain the preferential affinity of FcDFR1 for DHK as substrate. To prove this, another methodology is necessary.

Finally, from all these in silico analysis it can be hypothesized that when a specific DFR (FcDFR1 or FcDFR2) reduces a specific substrate (DHQ or DHK) different phenolic colored pigments can be acumulated in the white strawberry fruit.

\section{References (mandatory)}

Beld M., Martin C., Huits H., Stuitje A.R., Gerats A.G. (1989) Plant Mol. Biol. 13, 491-502.

Johnson E.T., Ryu S., Yi H., Shin B., Cheong H., Choi G. (2001) Plant J. 25, 325-333.

Johnson E.T., Yi H., Shin B., Oh B.J., Cheong H., Choi G. (1999) Plant J. 19, 81-85.

Miosic S., Thill J., Milosevic M., Gosch C., Pober S., Molitor C., Ejaz S., Rompel A., Stich K., Halbwirth H. (2014) PloS One, 9(11), e1 12707. 
Petit P., Granier T., d'Estaintot B. L., Manigand C., Bathany K., Schmitte, J. M., Lauvergeat V., Hamdi S., Gallois B. (2007) J. Mol. Biol. 368, 1345-1357.

Schwinn K., Miosic S., Davies K., Thill J., Gotame T. P., Stich K., Halbwirth H. (2014) Planta, 240, 1003-1010.

Simirgiotis, M. J., \& Schmeda-Hirschmann, G. (2010). J. Food Comp. Anal. 23(6), 545-553. 